\title{
DESIGN CONCEPT FOR AGS INJECTION KICKER UPGRADE TO 2 GEV*
}

\author{
R.B. Armenta, M.J. Barnes, E.W Blackmore, O. Hadary, G.D. Wait, TRIUMF, Vancouver, B.C., \\ L. Ahrens, C.J. Gardner, W. Zhang, Brookhaven National Laboratory, Upton, NY
}

\section{Abstract}

The present AGS injection kickers at A5 location were designed for $1.5 \mathrm{GeV}$ proton injection. Recent high intensity runs have pushed the transfer kinetic energy to $1.94 \mathrm{GeV}$, but with an imperfect matching in transverse phase space. Space charge forces result in both fast and slow beam size growth and beam loss as the size exceeds the AGS aperture. A proposed increase in the AGS injection energy to $2 \mathrm{GeV}$ with adequate kick strength would greatly reduce the beam losses making it possible increase the intensity from $70 \mathrm{TP}\left(70 * 10^{12}\right.$ protons/s) to 100 TP. R\&D studies are being undertaken by TRIUMF, in collaboration with BNL, to design two new kicker magnets for the AGS A10 location which will provide an additional deflection of $1.5 \mathrm{mrad}$ for $2 \mathrm{GeV}$ protons. TRIUMF has proposed a design for a $12.5 \Omega$ transmission line kicker magnet with rise and fall times of $100 \mathrm{~ns}, 3 \%$ to $97 \%$, and kick strength uniformity for protons of $\pm 3 \%$ over $90 \%$ of the aperture, powered by matched $12.5 \Omega$ pulse-forming lines. This paper describes the present status of a prototype design including the results of detailed 2D and 3D electromagnetic modelling of a transmission line kicker magnet and PSpice time domain analysis of the magnetic kick strength.

\section{INTRODUCTION}

Table 1:Existing A5 and proposed A10 kicker parameters.

\begin{tabular}{|l|l|l|}
\hline Parameter & Existing A5 & Proposed A10 \\
\hline Proton energy & $2.0 \mathrm{GeV}$ & $2.0 \mathrm{GeV}$ \\
\hline Deflection angle & $2.48 \mathrm{mrad}$ & $1.5 \mathrm{mrad}$ \\
\hline Gap height & $57.15 \mathrm{~mm}$ & $81 \mathrm{~mm}$ \\
\hline Gap width & $127 \mathrm{~mm}$ & $137 \mathrm{~mm}$ \\
\hline Rise time 3 to $97 \%$ & $140 \mathrm{~ns}$ & $100 \mathrm{~ns}$ \\
\hline Fall time 97\% to 3\% & $140 \mathrm{~ns}$ & $100 \mathrm{~ns}$ \\
\hline Flat-top Duration & 0 to $2250 \mathrm{~ns}$ & 0 to $1650 \mathrm{~ns}$ \\
\hline Pulse Repetition & $7.5 \mathrm{~Hz}$ & $7.5 \mathrm{~Hz}$ \\
\hline Number of magnets & 3 & 2 \\
\hline Total magnet length & $0.953 \mathrm{~m}$ & $0.66 \mathrm{~m}$ \\
\hline Magnetic field & $24.2 \mathrm{mT}$ & $19.1 \mathrm{mT}$ \\
\hline Current & $1100 \mathrm{~A}$ & $1235 \mathrm{~A}$ \\
\hline PFN Voltage & $32.5 \mathrm{kV}$ & $30.9 \mathrm{kV}$ \\
\hline Magnet type & lumped & $\begin{array}{l}\text { transmission } \\
\text { line }\end{array}$ \\
\hline
\end{tabular}

Three existing lumped inductance A5 injection kickers were designed for $1.5 \mathrm{GeV}$ proton injection. Adding two kickers, located in the A10 section, approximately $17 \mathrm{~m}$ downstream of A5, will increase the total beam deflection (Table 1).

\footnotetext{
* Work supported by a contribution from the Canada Foundation for Innovation.
}

\section{MAGNET DESIGN AND CONSTRUCTION}

\section{General}

A carefully matched high bandwidth system is needed to obtain the stringent pulse response requirements. Hence each of the two A10 systems is composed of a Pulse Forming Line (PFL) and an 11 cell travelling wave kicker magnet, connected by a matched transmission line and terminated by a matched resistor. To obtain a compact and cost efficient design a low system impedance $(Z)$ of $12.5 \Omega$ is chosen. Fig 1 gives the basic circuit diagram.

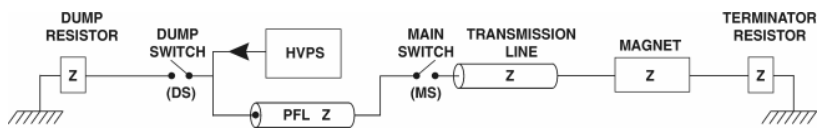

Figure 1: Schematic circuit of system.

The PFL nominal operating voltage is $31 \mathrm{kV}$. BNL requires that the system be tested at $50 \%$ above its nominal voltage, i.e. $46.5 \mathrm{kV}$. A design voltage of $50 \mathrm{kV}$ allows the use of several proven components such as transmission line, connectors and terminator resistors [1].

\section{Construction}

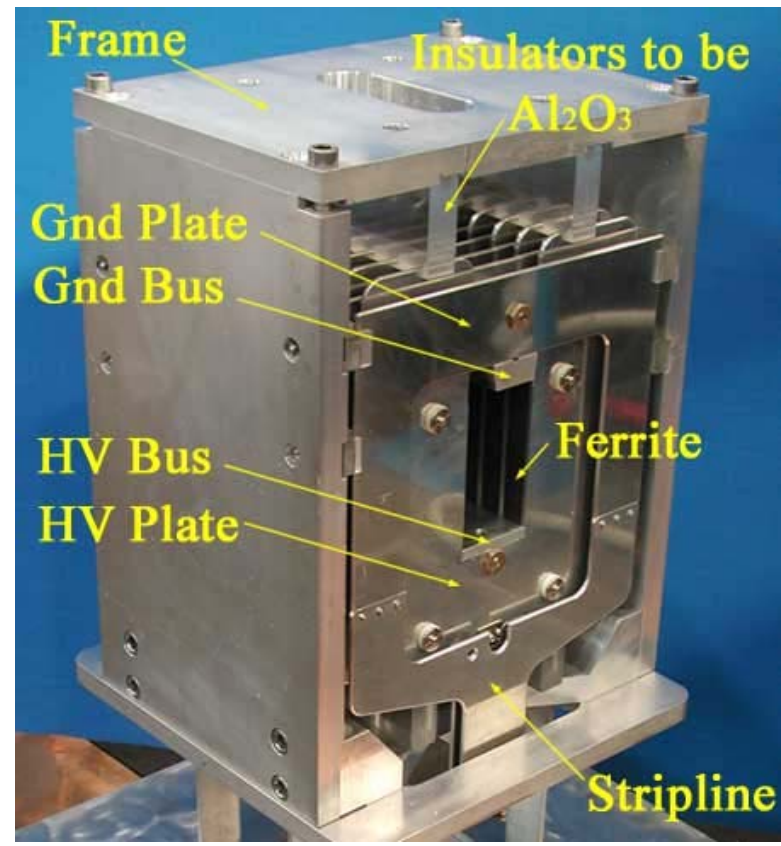

Figure 2: Prototype TRIUMF Kicker Magnet.

The proposed design for the AGS A10, 11 cell, transmission line kicker, is based on a TRIUMF prototype 10 cell kicker [2,3] (Fig 2), which was based on designs originated at CERN [4]. The A10 kickers will be fabricated using 304L Stainless Steel. This is due to requirements of both vacuum (the AGS vacuum is 
typically in the range $10^{-7}$ Torr to $10^{-9}$ Torr) and bake out at $200^{\circ} \mathrm{C}$. The transmission line type kicker consists of ferrite $\mathrm{C}$-core sections sandwiched between high voltage capacitance plates. One C-core, together with its ground and high voltage capacitance plates, is termed a cell. Each cell conceptually begins and ends in the middle of the HV capacitance plates.

The 11 cell A10 kicker has two main stainless steel conductors: a high voltage (HV) busbar and a ground (GND) busbar. The HV busbar interconnects twelve HV capacitance plates, and the GND busbar interconnects thirteen GND plates. The injected AGS proton beam will travel through the aperture and encounter magnetic and electric fields. Careful design of the mechanical components (distances and radii) aims to maintain the maximum electrical field below $5 \mathrm{kV} / \mathrm{mm}[8]$.

\section{Kicker Magnet Impedance}

The characteristic impedance is given by $Z=\sqrt{L / C}$, where $L$ and $C$ are the inductance and capacitance per cell of the kicker magnet, respectively. Reflections cause field ripple and potentially reduce the life of the thyratron switches. In order to avoid impedance mismatches, and therefore reflections, the impedance of the kicker magnet must closely match the impedance of the system. The total series inductance of a central cell is determined by the aperture dimensions and can reasonably accurately be calculated from the equation: $L=\mu_{0} \times w \times l / h$, $(63.89 \mathrm{nH})$ where, $w$ is the width of the aperture ( $137 \mathrm{~mm}$ for optimized aperture), $l$ is the length of a cell $(30.06 \mathrm{~mm})$, and $h$ is the height of the aperture $(81 \mathrm{~mm})$. However, since the legs of the ferrite are profiled (Fig 3) to improve the deflection uniformity, it is necessary to use a code such as Opera2D [5] to accurately calculate the total series inductance of a cell: simulations give $62.36 \mathrm{nH}$ per central cell. Thus, to achieve a $12.5 \Omega$ kicker magnet, the nominal cell capacitance is $399.1 \mathrm{pF}$.

The capacitance of a cell is made up of several terms including parallel plate capacitance between the HV and GND plates, and edge effects between the sides of the HV plates and both the GND plates as well as the magnet frame. To achieve the $399 \mathrm{pF}$ capacitance with a high degree of accuracy several cells of the kicker magnet have been simulated [6] with the 3D electromagnetic software Coulomb [7]. In order to validate Coulomb, the software was used to model the prototype TRIUMF kicker magnet and the predicted capacitance was compared with measurements [8].

\section{Deflection Uniformity}

The specifications for the AGS A10 kicker magnet require an aperture width (the distance between the adjacent surfaces of the HV busbar and the GND busbar) of $127 \mathrm{~mm}$, and an aperture height (the distance between the inner surfaces of the legs of the C-core ferrite) of $81 \mathrm{~mm}$. However 2D electromagnetic simulations carried out to optimize field uniformity[12] within the AGS A10 aperture resulted in the following (Fig 3):
- Increasing the aperture width to $137 \mathrm{~mm}$;

- Shaping of the aperture side surface of the GND busbar (this surface of the GND busbar was flat for the prototype TRIUMF kicker magnet);

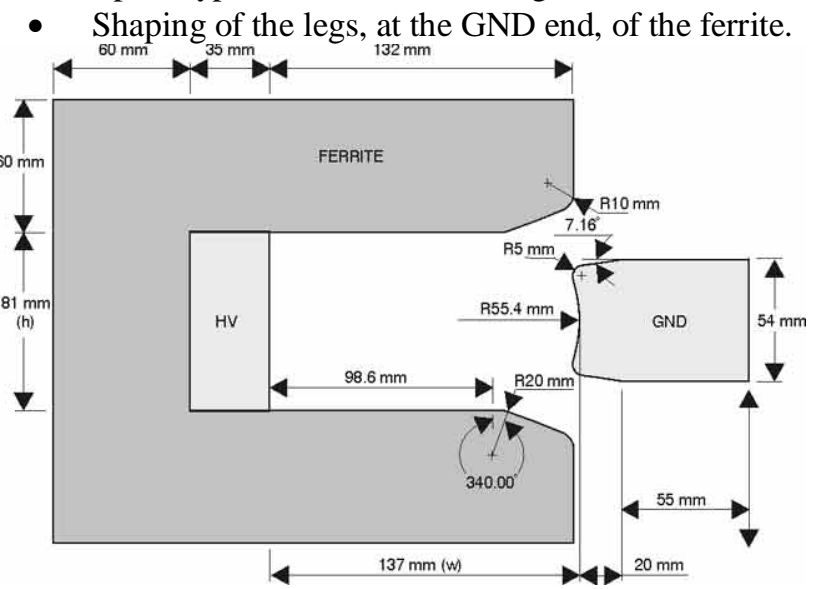

Figure 3: Cross section of optimized aperture.

The total deflection angle is obtained from the vector addition of the deflection attributable to the horizontal magnetic field and the vertical electric field. The magnetic and electric components of deflection are arranged to be added for the optimized geometry, giving the most uniform deflection. Fig 4 shows a contour plot of the resultant total deflection for protons, normalized to the deflection at the centre of the aperture. The uniformity is within $\pm 1 \%$ over $85 \%$ of the aperture area and within $\pm 2.1 \%$ over $90 \%$ of the aperture area. Since $\beta$ is smaller for $\mathrm{Au}^{77+}$ ions than for protons, the electric field kick is larger for $\mathrm{Au}$ than for protons: for $\mathrm{Au}^{77+}$ the uniformity is within $\pm 3 \%$ over $80 \%$ of the aperture area.

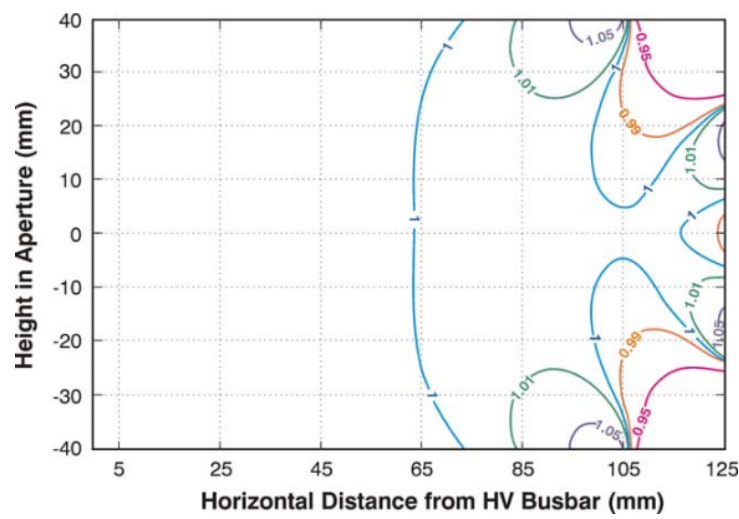

Figure 4: Normalized contour plot of $\theta_{x}=\theta_{B, x}+\theta_{E, y}$, for protons for the optimized geometry.

Similar deflection uniformity could also be achieved without having to profile the end of the legs of the ferrite or the ground busbar. However the separation of the GND and HV busbars would need to be $147 \mathrm{~mm}$ resulting in a predicted inductance of $68.9 \mathrm{nH} / \mathrm{cell}, 10.5 \%$ greater than the geometry of Fig 3. Hence, to achieve $12.5 \Omega$ impedance, the nominal cell capacitance would need to be increased by $10.5 \%$, therefore increasing the size and cost of the kicker as well as the cell transit time. $(\sqrt{L \times C})$ 


\section{PSpice Modelling}

The electrical circuit of the kicker system, including PFL, thyratrons, transmission line, kicker magnet and terminators has been optimised using PSpice [8]. The model of the thyratrons is based on a previous model, which includes displacement current [9], but has been modified to simulate the decay of the plasma density at the end of the current pulse. The plasma density drops with a time constant in the region of $2 \mu$ s to $7 \mu$ s [10]: analysis of previous measurements [11] show a time constant of $5 \mu$ s for a CX2003, 3 stage, thyratron: the $5 \mu \mathrm{s}$ is used in the revised model.

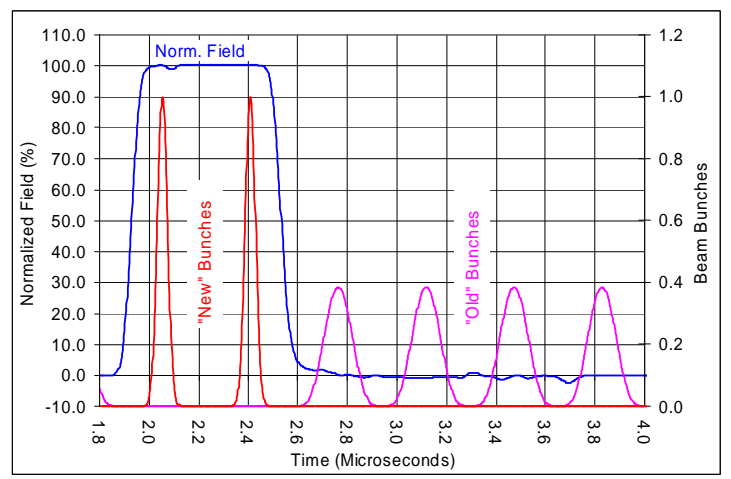

Figure 5: Predicted Field Pulse, and simulated "Beam Bunches" for 2 bunches per transfer.

Measurements and simulations for the prototype TRIUMF kicker [3] show the validity of using Opera3D, to calculate the inductance of all the cells of a kicker, including fringe fields at the ends. The same procedure has been applied to the AGS A10 kicker magnets and the calculated cell inductance has been included in a revised mathematical model of the kicker magnet: the detailed results, and the revised model, will be presented at MT19.

The PSpice model also includes dispersion in the PFLs and the transmission cables: the frequency dependent dispersion characteristics have been fitted to the cable manufacturers data sheets. The simulations include a representation of the beam bunches, to allow optimization of the field. The next-to-last bunches and bunches previously transferred into the AGS ring are diluted on the AGS injection porch: these are referred to here as "Old" beam bunches. "New" beam bunches are those that have recently been transferred from the AGS Booster. Fig 5 shows the predicted kicker field pulse, as well as the representation of the beam bunches, for the transfer of 2 bunches from the Booster to AGS ring per injection cycle. The effect of the length of the transmission cable between the PFL and the kicker magnets has been studied, within constraints of the site ( 250 feet minimum from PFL to kicker), to minimize the deflection of "old" bunches due to post pulse ripple. The predicted $3 \%$ to $97 \%$ rise time is $97 \mathrm{~ns}$, which is within the specified $100 \mathrm{~ns}$.

\section{SUMMARY}

A design has been carried out for the proposed AGS A10 kicker system. The aperture of the kicker magnet has been optimized to achieve the required beam deflection uniformity, for proton transfer, while minimizing cell inductance. The plate geometry to achieve the cell capacitance has been selected based on detailed 3D electromagnetic simulations: the software has been validated against measurements on a transmission line kicker magnet. The kicker impedance is expected to be correct and hence is not designed to be adjustable. PSpice time domain analysis of the magnetic kick strength has been carried out to optimize the overall system.

\section{ACKNOWLEDGEMENT}

The authors acknowledge the extensive mechanical design work carried out by Tim Emmens.

\section{REFERENCES}

[1] L. Ducimetière, N. Garrel, M.J. Barnes, G.D. Wait, "The LHC Injection Kicker Magnet", PAC 2003, Portland Oregon, pp1162-1164.

[2] G.D. Wait, M.J. Barnes and H.J. Tran, "Magnetic Field in a Prototype Kicker Magnet for the Kaon Factory", IEEE Trans. on Magnetics, July 1994, Vol. 30, No. 4, pp2118-2121.

[3] M.J. Barnes, G.D. Wait, "Comparison of Measured and Predicted Inductance per Cell for a Travelling Wave Kicker Magnet”, EPAC, Sitges, Spain, 1996, pp2588-2590.

[4] Fiander D., Metzmacher K. and Pearce P., "Kickers and Septa at the PS Complex, CERN". Kaon PDS Magnet Design Workshop, Oct. 1988, pp71-79.

[5] Vector Fields Inc., 1700 North Farnsworth Av., Aurora, IL 60505, USA, www.vectorfields.com

[6] Ori Hadary "Alternating Gradient Synchrotron Kicker Magnet Simulation Results", ENGR 446 Report, January 1, 2005.

[7] Integrated Engineering Software, Winnipeg, Manitoba, Canada, www.integratedsoft.com.

[8] M.J. Barnes and G.D. Wait, "PSpice Analysis of the AGS A10 Kicker Magnets", TRI-DN-05-15, May 2005.

[9] M.J. Barnes, G.D. Wait, "A mathematical model of a three-gap thyratron simulating turn-on", Pulse Power Conf., Albuquerque, New Mexico, June 1993, pp293296.

[10]E2V Technologies Ltd, "Hydrogen Thyratrons Preamble", Issue dated September 2002, http://www.e2v.com/datasheets/thyratrons/thyratron preamble.pdf

[11] M.J. Barnes and G.D. Wait, E. Carlier, L. Ducimetière, G.H. Schröder, E.B. Vossenberg, "High Voltage Measurements On A Prototype PFN For The LHC Injection Kickers", PAC99, New York, March 29-April 2, 1999, pp1509-1511.

[12]R.B. Armenta, M.J. Barnes, O. Hadary, G.D. Wait, "AGS A10 Injection Kicker Development: Deflection Angle Uniformity And Cell Inductance Based On A Two Dimensional Model Of The Optimized Geometry", TRI-DN-05-05, February 21, 2005. 